\title{
Recovery of BMIPP Uptake and Regional Wall Motion in Insulin Resistant Patients Following Angioplasty for Acute Myocardial Infarction
}

\author{
Takayuki Fujino, MD*,**; Yoshinao Ishii, MD*; Toshiharu Takeuchi, MD**; \\ Kunihiko Hirasawa, MD*; Kunihiko Tateda, MD*; \\ Kenjiro Kikuchi, MD**; Naoyuki Hasebe, MD**
}

\begin{abstract}
The effect of insulin resistance (IR) on the fatty acid metabolism of myocardium, and therefore on the recovery of left ventricular (LV) wall motion, has not been established in patients with acute myocardial infarction (AMI). A total of consecutive 58 non-diabetic AMI patients who had successfully undergone emergency coronary angioplasty were analyzed retrospectively. They were categorized into 2 groups, normal glucose tolerance (NGT) and impaired glucose tolerance (IGT), based on a 75-g oral glucose tolerance test (OGTT). The parameters of OGTT, myocardial scintigraphy $(\mathrm{n}=58)$ (thallium-201 (Tl) and iodine- $123 \beta$-methyl-iodophenylpentadecanoic acid (BMIPP)) and left ventriculography $(\mathrm{n}=24)$ were compared in the 2 groups after reperfusion (acute phase) and 3-4 weeks after the AMI (chronic phase). The insulin resistance (IR), estimated by the serum concentration of insulin at $120 \mathrm{~min}$ (IRI 120') of the OGTT and by the HOMA (the homeostasis model assessment) index, was higher in the IGT group than in NGT group. An inverse correlation was found between the recovery of regional LV wall motion in the ischemic lesion and the IRI 120' and HOMA index. Although the recovery of BMIPP uptake from the acute to the chronic phase was higher in the IGT group, it was only correlated with the degree of IRI 120', not with the HOMA. IR accompanied by IGT can negatively influence the recovery of regional LV wall motion. (Circ J 2003; 67: 757-762)
\end{abstract}

Key Words: Acute myocardial infarction; Fatty acid metabolism; Insulin resistance; Wall motion abnormality

$\mathbf{M}$ any studies have shown the relationships between the incidence of cardiovascular disease (CVD), its mortality, impaired glucose tolerance (IGT) and insulin resistance (IR)!-5 Glucose intolerance can induce abnormalities in cardiac function not only through abnormal myocardial blood flow, but also through metabolic abnormalities in the myocardium, known as "diabetic cardiomyopathy'6,7 In patients with mild glucose intolerance, abnormal $\beta$-oxidation of myocardial fatty acid may induce abnormal cardiac function; ${ }^{8}$ however, that same research group subsequently reported normal fluoro-heptadecanoic acid uptake in the heart of subjects with impaired glucose tolerance, which suggests that the concept of alterations in myocardial fatty acid metabolism remains controversial.

Direct percutaneous transluminal coronary angioplasty (PTCA) is widely accepted as part of the treatment of acute myocardial infarction (AMI), based on the excellent results from several randomized trials ${ }^{10}$ Patients with AMI and diabetes mellitus (DM) have a much poorer prognosis than non-diabetic patients 11,12 Although there are no differences in the recovery of coronary flow after angioplasty and the

(Received January 24, 2003; revised manuscript received June 25, 2003; accepted July 2, 2003)

*The Division of Internal Medicine, Asahikawa City Hospital and **The First Department of Internal Medicine, Asahikawa Medical College, Asahikawa, Japan

Mailing address: Takayuki Fujino, MD, The First Department of Internal Medicine, Asahikawa Medical College, 2-1-1-1 Midorigaoka Higashi, Asahikawa 078-8510, Japan. E-mail: ffuji42@asahikawamed.ac.jp infarct size, there is great variability in cardiac function following infarction, especially in patients with DM, which is probably related to reperfusion injury? Several factors had been reported as involved in reperfusion injury and different therapies have been used: glucose-insulinpotassium infusion therapy, and infusion of nicorandil, an adenosine triphosphate (ATP)-sensitive $\mathrm{K}^{+}$channel opener ${ }^{13-15}$

There are few reports concerning the effects of IGT on the clinical outcome and whether there are changes in myocardial fatty acid metabolism and left ventricular (LV) wall motion of the IR patient who has had an AMI treated by reperfusion therapy. We used myocardial scintigraphy to investigate the effects of IGT and IR on the recovery of LV regional wall motion (RWM), blood flow, and fatty acid metabolism in such patients.

\section{Methods}

We restropectively analyzed consecutive Japanese patients with an AMI between April 1996 and November 1999, who underwent emergency coronary angiography and demonstrated total occlusion or $99 \%$ stenosis of a coronary artery with delayed flow (TIMI grade 0 or 1$) !^{16}$ All of the patients underwent PTCA, with complete recanalization of the occluded artery (TIMI grade 3 ). Based on the response to a $75-\mathrm{g}$ oral glucose tolerance test (OGTT) ${ }^{17}$ performed 3 weeks after the onset of the AMI, 58 patients were categorized into 2 groups: normal glucose tolerance test (NGT), and impaired glucose tolerance (IGT). Patients receiving drug therapy for DM were excluded from this 
study. We evaluated the degree of IR based on the insulin concentration at $120 \mathrm{~min}$ after glucose administration, ${ }^{18}$ and by the homeostasis model assessment $(\mathrm{HOMA}=$ (fasting blood sugar $(\mathrm{FBS}, \mathrm{mg} / \mathrm{dl}) / 18) \times($ IRI 0' $(\mu \mathrm{U} / \mathrm{ml}) / 22.5)$; IRI 0 , blood immunoreactive insulin concentration immediately after glucose administration) ${ }^{19}$ The HOMA index is thought to reflect hepatic IR, whereas the IRI 120' reflects peripheral IR ${ }^{20}$ but the degree of IR is not the combination of the HOMA and IRI 120' as they are separate measures of IR. Written informed consent was obtained from each patient and the study protocol was approved by the institutional Ethics Committee.

\section{Estimation of Myocardial Blood Flow and Fatty Acid Metabolism By Myocardial Scintigraphy}

To estimate the recovery of myocardial blood flow and fatty acid metabolism after PTCA, all 58 patients underwent myocardial scintigraphy using thallium-201 (Tl) at 3-4 days after the onset of AMI (acute phase), and iodine-123$\beta$-methyliodophenylpentadecanoic acid (BMIPP) at both 5-7 days (acute phase), and at both 3 weeks after the onset (chronic phase; BMIPP and Tl single photon emission computed tomography (SPECT) imaging were performed on alternate days). While at rest, the patients underwent BMIPP and Tl SPECT imaging using a rotating gamma camera equipped with a low-energy, general-purpose collimator (Toshiba, Japan). At rest after fasting, approximately $12 \mathrm{~h}$ after the last meal, $111 \mathrm{MBq}$ of BMIPP were injected and SPECT imaging was started 30 min later. We collected 32 views over 180 degrees from the right anterior oblique (RAO) to the left posterior oblique (LAO) positions at $30-40$ s per view. For the Tl scintigraphy, $10-15$ min after injection of $100-130 \mathrm{MBq} \mathrm{Tl}$ while the patient was at rest, 32 views of $30 \mathrm{~s}$ each were collected. In each SPECT study, a series of transaxial images were reconstructed using filtered backprojection, after which cardiac short-axis and long-axis slices perpendicular to the cardiac axis were identified.

For the qualitative SPECT data analysis, 2 short-axis slices and 1 vertical long-axis slice were selected from both the $\mathrm{Tl}$ and BMIPP studies as described previously? ${ }^{21}$ The 2 short-axis slices from the cardiac base and the mid- to -apical portion were divided into 8 segments each, and the apex from the vertical long-axis image was divided into 2 segments (Fig 1A). In total, 18 segments were scored based on the consensus of 2 experienced observers using a 4point grading system $(0=$ normal, $1=$ mildly reduced, $2=$ moderately reduced, $3=$ severely reduced or absent uptake). The sum of the defect scores for each segment is expressed as the total defect score (TDS), which was used to estimate the rate of recovery of tracer uptake from the acute to the chronic phase ((TDS in acute phase-TDS in chronic phase) /TDS in acute phase) $\times 100$, and is expressed as \% Tl TDS and \%BMIPP TDS. The Tl TDS to BMIPP TDS ratio (Tl/ $\mathrm{BM}), \mathrm{BMIPP}$ TDS minus Tl TDS (BM-Tl) and $\triangle \mathrm{BM}-\mathrm{Tl}$ ((BM-Tl in acute phase) minus (BM-Tl in chronic phase)) were calculated. Evaluation of the imaging studies was blinded and the images were read by 2 experienced physicians who were unaware of the patient's clinical history or the coronary angiography or ECG findings. The agreement between the 2 observers was $91 \%$ for the BMIPP images and $87 \%$ for the $\mathrm{Tl}$ images.

Estimation of Cardiac Function and Regional Wall Motion by Left Ventriculography

To estimate LV function, patients underwent left ven- triculography (LVG) just after successful emergency PTCA (acute phase) and at 4 weeks after the onset of AMI (chronic phase). As the indices of $\mathrm{LV}$ function, the $\mathrm{LV}$ ejection fraction (LVEF) and the LV end-diastolic volume index (LVEDVI) and end-systolic volume index (LVESVI) were analyzed by the area-length method,2 using the RAO views of the left ventriculogram. The recovery rates for LVEF, LVEDVI, and LVESVI from the acute to the chronic phase were calculated and expressed as \%LVEF, \%LVEDVI, and \%LVESVI ((LVEF, LVEDVI, LVESVI in chronic phase-LVEF, LVEDVI, LVESVI in acute phase) /LVEF, LVEDVI, LVESVI in chronic phase) $\times 100$ ).

To estimate the LV RWM, we analyzed the LVG images by the centerline method using 100 chords (Fig 2a). Normalized motion in each chord is expressed as the standard deviation from the mean value for normal individuals (SD/ chord) 23 To assess the severity of hypokinesis in the LV territory supplied by the occluded artery, the territories supplied by the left anterior descending branch and right coronary arteries (LAD and RCA) extend from chords $10-66$ for the LAD and from 51 to 80 for the RCA, in the 30 -degree RAO projection (Fig $2 \mathrm{a}$ ) ${ }^{23}$ We calculated the sum of the SD/chord for each territory. In addition, the recovery rate of the sum of the $\mathrm{SD} /$ chord values from the acute to the chronic phase was calculated and expressed as $\% \mathrm{SD} /$ chord ((the sum of SD/chord in acute phase-the sum of $\mathrm{SD} /$ chord in chronic phase)/the sum of SD/chord in acute phase $) \times 100$ ). We excluded the data from subjects with left circumflex artery (LCX) territories supplied by the occluded artery because that SD/chord has been shown to have a great variability. ${ }^{3}$ We used the LVG data of 24 subjects.

Total cholesterol (TC), triglyceride (TG), high density lipoprotein-cholesterol (HDL-C), and free fatty acid (FFA) concentrations were determined by enzymatic methods. HemoglobinA1c, atrial natriuretic peptide (ANP) and brain natriuretic peptide (BNP) concentrations were also estimated 3 weeks after AMI.

\section{Statistical Analysis}

All data are expressed as the mean \pm SD. The MannWhitney test was used to compare 2 groups. Chi-squared analysis was used to compare discrete variables between the 2 groups. A value of $\mathrm{p}<0.05$ was considered statistically significant. Linear regression analysis was used to determine the relationship between 2 variables and multiple regression analysis was used to determine the relationship between multiple variables.

\section{Results}

The clinical characteristics of the 2 groups did not differ significantly with respect to age, gender, presence of hypertension, hyperlipidemia, or smoking, the interval between the time of onset of chest pain and the time of reperfusion, or the peak creatine kinase activity (Table 1 ). There were no differences between the 2 groups in the extent of coronary artery disease, history of antecedent angina, intermittent claudication or stroke, the types of oral therapies for $\mathrm{CVD}$, or in the degree of mitral valve regurgitation estimated by LVG and ultrasound cardiography (UCG) (data not shown). There were no differences in TIMI grade or collateral flow grade (Rentrop grading) between NGT and IGT before reperfusion therapy (data not shown). In $38 \%$ of the subjects there was stent replacement following the 
Table 1 Clinical Characteristics

\begin{tabular}{|c|c|c|c|}
\hline & $\begin{array}{l}\text { NGT group } \\
\quad(n=30)\end{array}$ & $\begin{array}{l}\text { IGT group } \\
\quad(n=28)\end{array}$ & $p$ value \\
\hline Age (years) & $61.9 \pm 9.4$ & $60.7 \pm 9.2$ & $N S$ \\
\hline$M / F$ & $26 / 4$ & $25 / 3$ & $N S$ \\
\hline $\begin{array}{l}\text { Infarcted area } \\
\text { ant/inf/lat }\end{array}$ & \multicolumn{2}{|c|}{ Infarcted area } & $N S$ \\
\hline \multicolumn{4}{|l|}{ Coronary risk factors } \\
\hline Hypertension & $15(50 \%)$ & $14(50 \%)$ & $N S$ \\
\hline Smoking & $28(93 \%)$ & $27(96 \%)$ & $N S$ \\
\hline Hyperlipidemia & $10(30 \%)$ & $11(39 \%)$ & NS \\
\hline \multicolumn{4}{|c|}{ Interval between onset of infarction and reperfusion ( $\mathrm{min}$ ) } \\
\hline & $251 \pm 152$ & $241 \pm 114$ & NS \\
\hline Peak CK activity $(I U / L)$ & $4,205 \pm 1,635$ & $4,142 \pm 3,468$ & $N S$ \\
\hline
\end{tabular}

CK, creatine kinase; IGT, impaired glucose tolerance; NGT, normal glucose tolerance; ant, anterior; inf, inferior; lat, lateral. Data are expressed as the mean $\pm S D$.

Table 2 Laboratory Parameters

\begin{tabular}{lccc}
\hline \hline & $\begin{array}{c}\text { NGT group } \\
(n=30)\end{array}$ & $\begin{array}{c}\text { IGT group } \\
(n=28)\end{array}$ & p value \\
\hline Total cholesterol $(\mathrm{mg} / \mathrm{dl})$ & $202 \pm 34$ & $204 \pm 37$ & $\mathrm{NS}$ \\
HDL cholesterol $(\mathrm{mg} / \mathrm{dl})$ & $40 \pm 12$ & $37 \pm 6$ & $\mathrm{NS}$ \\
Triglyceride $(\mathrm{mg} / \mathrm{dl})$ & $138 \pm 62$ & $146 \pm 63$ & $\mathrm{NS}$ \\
LDL cholesterol $(\mathrm{mg} / \mathrm{dl})$ & $133 \pm 33$ & $138 \pm 35$ & $\mathrm{NS}$ \\
FFA $(\mathrm{mEq} / \mathrm{L})$ & $0.36 \pm 0.20$ & $0.39 \pm 0.12$ & $\mathrm{NS}$ \\
ANP $(\mathrm{pg} / \mathrm{ml})$ & $31 \pm 25$ & $33 \pm 34$ & $\mathrm{NS}$ \\
BNP $(\mathrm{pg} / \mathrm{ml})$ & $108 \pm 90$ & $61 \pm 62$ & $\mathrm{NS}$
\end{tabular}

$A N P$, atrial natriuretic peptide; BNP, brain natriuretic peptide; FFA, free fatty acid; NGT, normal glucose tolerance; IGT, impaired glucose tolerance. Data are expressed as the mean $\pm S D$.

PTCA for AMI, but there was no difference between the PTCA alone group and PTCA followed by stent replacement group in the parameters of glucose tolerance and IR (data not shown).

There were no significant differences between the 2 groups in the lipid concentrations or in the concentrations of ANP and BNP (Table2). The FBS, $\Sigma$ BS, body mass index (BMI) and HbA1c were higher in the IGT group than in the NGT group (Table 3 ), but the IRI 0' and $\Sigma$ IRI were not different between the 2 groups. The HOMA and IRI $120^{\prime}$, as indices of IR ${ }^{24}$ were higher in the IGT group than in the NGT group (Table 3 ).

When compared between the IGT and NGT groups, the TDS of Tl and BMIPP were not different in either the acute or the chronic phase (Table 4). The Tl TDS to BMIPP TDS ratio $(\mathrm{Tl} / \mathrm{BM})$ in the acute phase was lower in the IGT group than in the NGT group, but was similar between the groups in the chronic phase (Table4). The $\mathrm{BM}-\mathrm{Tl}$ in the acute phase and $\triangle \mathrm{BM}-\mathrm{Tl}$ were higher in the IGT group than in the NGT group. When compared between the acute and chronic phases, the TDS of Tl and BMIPP was significantly decreased in the chronic phase (Table4). In the IGT group, the Tl/BM in the chronic phase was increased compared with the acute phase, but not in the NGT group in which the Tl/BM was not significantly different between the acute and chronic phases (Table 4). The \% Tl TDS was similar in the 2 groups (Table 4); however, the \%BMIPP TDS in the IGT group was greater than in the NGT group (Table 4). To estimate the recovery of BMIPP and Tl uptake in the risk area of the occluded artery, the regional defect score (RDS) was calculated $(1,5-9,13-18$ for LAD;
Table 3 Laboratory and Oral Glucose Tolerance Test Parameters

\begin{tabular}{lccc}
\hline \hline & $\begin{array}{c}\text { NGT group } \\
(n=30)\end{array}$ & $\begin{array}{c}\text { IGT group } \\
(n=28)\end{array}$ & p value \\
\hline FBS $(\mathrm{mg} / \mathrm{dl})$ & $88 \pm 7$ & $99 \pm 12$ & $<0.05$ \\
HbAlc $(\%)$ & $5.4 \pm 0.5$ & $5.7 \pm 0.7$ & $<0.05$ \\
$B M I\left(\mathrm{~kg} / \mathrm{cm}^{-2}\right)$ & $22.0 \pm 3.1$ & $24.5 \pm 2.5$ & $<0.05$ \\
$\Sigma \mathrm{BS}$ & $653 \pm 105$ & $875 \pm 144$ & $<0.05$ \\
$I R I 0^{\prime}(\mu \mathrm{U} / \mathrm{ml})$ & $6.0 \pm 7.3$ & $6.3 \pm 1.8$ & $N S$ \\
$I R I 120^{\prime}(\mu \mathrm{U} / \mathrm{ml})$ & $49 \pm 45$ & $93 \pm 46$ & $<0.05$ \\
$\Sigma I R I$ & $278 \pm 119$ & $283 \pm 182$ & $N S$ \\
HOMA & $1.32 \pm 0.48$ & $1.52 \pm 0.50$ & $<0.05$ \\
\hline
\end{tabular}

BMI, body mass index; FBS, fasting blood sugar; HOMA, the homeostasis model assessment, (FBS (mg/dl) /18)* (IRI 0' (mU/ml) /22.5); IRI 0', 120', blood immunoreactive insulin concentration at 0 or 120 min after glucose administration; $\Sigma$ BS, $\Sigma$ IRI, the sum of blood sugar, IRI concentration at 0 , 30, 60, 90, and 120 min after glucose administration. NGT, normal glucose tolerance; IGT, impaired glucose tolerance. Data are expressed as the mean $\pm S D$.

Table 4 Parameters of Myocardial Scintigraphy

\begin{tabular}{lccc}
\hline \hline & $\begin{array}{c}\text { NGT group } \\
(n=30)\end{array}$ & $\begin{array}{c}\text { IGT group } \\
(n=28)\end{array}$ & p value \\
\hline Tl TDS (acute) & $12.4 \pm 6.9$ & $9.3 \pm 7.0$ & $N S$ \\
BMIPP TDS (acute) & $15.2 \pm 7.1$ & $14.9 \pm 8.4$ & $N S$ \\
BM-Tl TDS (acute) & $2.83 \pm 2.56$ & $5.50 \pm 3.48$ & $<0.05$ \\
Tl/BM TDS (acute) & $0.81 \pm 0.41$ & $0.62 \pm 0.31$ & $<0.05$ \\
Tl TDS (chronic) & $10.7 \pm 5.3$ & $8.1 \pm 6.2$ & $N S$ \\
BMIPP TDS (chronic) & $12.7 \pm 7.4$ & $11.2 \pm 7.6$ & $N S$ \\
BM-Tl TDS (chronic) & $2.03 \pm 3.34$ & $3.11 \pm 4.87$ & $N S$ \\
Tl/BM TDS (chronic) & $0.83 \pm 0.42$ & $0.74 \pm 0.36$ & $N S$ \\
\%Tl TDS & $11.2 \pm 26.2$ & $12.2 \pm 29.3$ & $N S$ \\
\%BMIPP TDS & $16.4 \pm 20.5$ & $24.8 \pm 16.7$ & $<0.05$ \\
$\triangle B M-T l$ TDS & $0.83 \pm 3.74$ & $2.39 \pm 3.60$ & $<0.05$ \\
\hline
\end{tabular}

Acute, acute phase (3-7 days); BMIPP, iodine-123ß -methyliodophenylpentadecanoic acid; chronic, chronic phase (3 week); TDS, total defect score; $T l$, thallium-201; \%Tl, \%BMIPP TDS, the recovery rate of the TDS for Tl, or BMIPP from the acute to chronic phase; $\triangle B M-T l T D S$, (BM-Tl TDS in acute) minus (BM-Tl TDS in chronic); NGT, normal glucose toler-ance; $I G T$, impaired glucose tolerance. Data are expressed as the mean $\pm S D$.

(A)

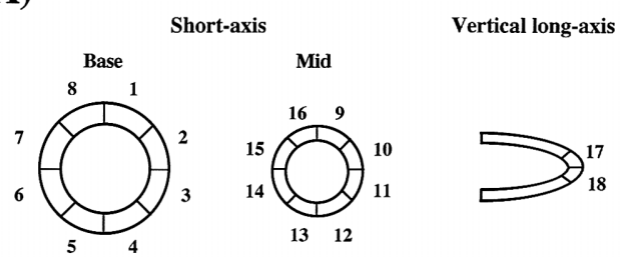

(B) $\mathbf{~ T l}$
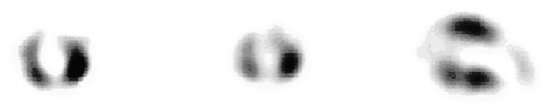

(C) BMIPP

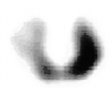

Fig 1. (A) Schematic representation of the left ventricular segments for single-photon-emission computed tomography (SPECT). Representative set of images showing Tl (B) and BMIPP (C) scintigraphy in a patient with acute myocardial infarction in the acute phase, and with left anterior descending branch (LAD) territories supplied by the occluded artery. 
Table 5 Left Ventriculography Parameters

\begin{tabular}{lccc}
\hline \hline & $\begin{array}{c}\text { NGT group } \\
(n=12)\end{array}$ & $\begin{array}{c}\text { IGT group } \\
(n=12)\end{array}$ & p value \\
\hline LVEDVI (acute) & $88 \pm 21$ & $87 \pm 17$ & $N S$ \\
LVEDVI (acute) & $34 \pm 16$ & $36 \pm 12$ & $N S$ \\
LVEF (acute) & $61 \pm 13$ & $58 \pm 12$ & $N S$ \\
SD/chord (acute) & $-2.3 \pm 0.6$ & $-1.9 \pm 0.8$ & $N S$ \\
LVEDVI (chronic) & $99 \pm 33$ & $82 \pm 17$ & $N S$ \\
LVESVI (chronic) & $37 \pm 15$ & $30 \pm 11$ & $N S$ \\
LVEF (chronic) & $61 \pm 10$ & $63 \pm 11$ & $N S$ \\
SD/chord (chronic) & $-1.8 \pm 0.7$ & $-1.7 \pm 0.7$ & $N S$ \\
$\%$ LVEDI & $9.7 \pm 20$ & $1.9 \pm 18$ & $N S$ \\
$\%$ LVESVI & $4.5 \pm 23$ & $0.7 \pm 25$ & $N S$ \\
$\%$ LVEF & $12.8 \pm 19$ & $3.0 \pm 13$ & $N S$ \\
$\% S D /$ chord & $20.5 \pm 27.4$ & $12.4 \pm 45.6$ & $N S$ \\
\hline
\end{tabular}

LVEDVI, left ventricular ( $L V)$ end-diastolic volume index, $\mathrm{ml} / \mathrm{m}^{2} ; L V E F, L V$ ejection fraction, \%; LVESVI, LV end-systolic volume index, $\mathrm{ml} / \mathrm{m}^{2} ; \mathrm{NGT}$, normal glucose tolerance; IGT, impaired glucose tolerance. Data are expressed as the mean $\pm S D$.

(A)

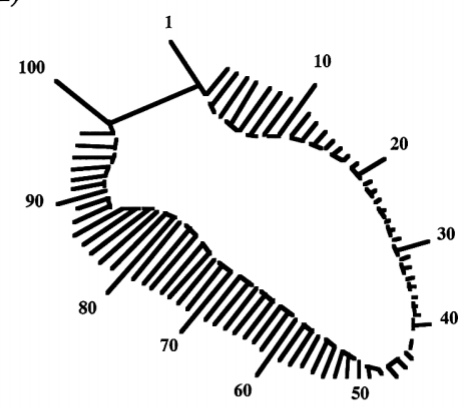

(B)

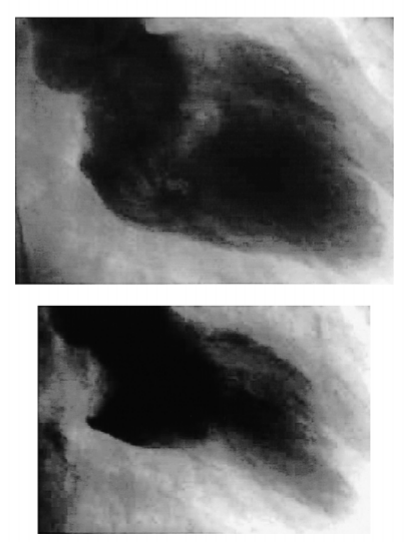

Fig 2. (A) Schematic representation of the left ventricular segments for SD/chord in left ventriculography. (B) Representative set of images showing left ventriculography in the diastolic (Upper image) and systolic (Lower image) phases in a patient with acute myocardial infarction just after successful emergency coronary angioplasty, and with left anterior descending branch (LAD) territories supplied by the occluded artery.

4, 5, 12, 13, 18 for RCA; 2, 3, 10, 11 for LCX in Fig 1A) as described previously.5 The RDS correlated significantly with the TDS $(r=0.95, p<0.01)$, suggesting that the improvement in BMIPP uptake can be attributed to improvement in the risk area of the occluded artery. The \%BMIPP RDS in the IGT group was greater than that of the NGT group, as was the \%BMIPP TDS (NGT, 16.8 \pm 20.1 ; IGT, 24.3 \pm 18.9 , $\mathrm{p}<0.05)$.

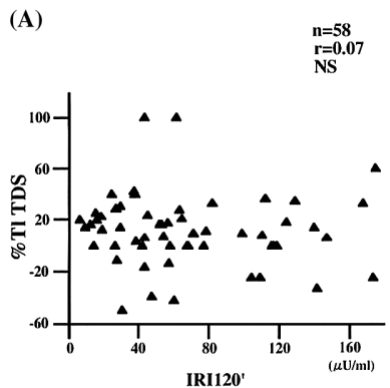

(B)

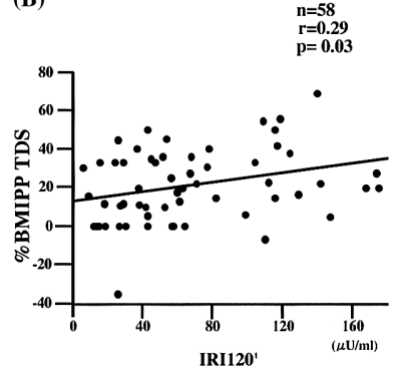

Fig 3. Relationship between IRI 120' and (A) \%Tl TDS and (B) $\%$ BMIPP TDS. IRI 120', blood immunoreactive insulin concentration 120 min after a 75 -g oral glucose test; \% Tl, \%BMIPP TDS, the recovery rate of the total defect score (TDS) for ${ }^{201} \mathrm{Tl}$, or ${ }^{123}$ I-BMIPP from the acute to the chronic phase.

(A)

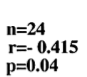

(B)

$\underset{\substack{r=0.0 .415 \\ p=0.04}}{ }$

$n=24$
$r=-0.435$
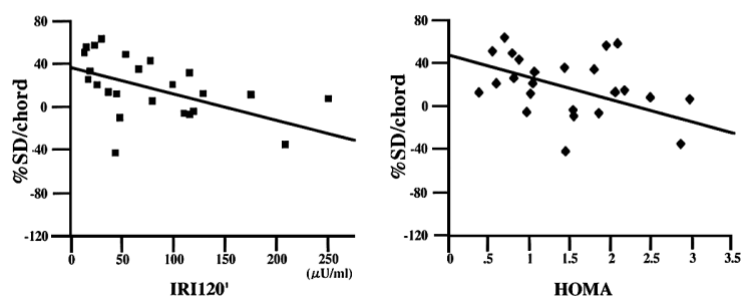

Fig 4. Relationship between \%SD/chord and (A) IRI 120' and (B) HOMA. IRI 120', serum immunoreactive insulin concentration at 120 min after a 75 -g oral glucose test $(\mu \mathrm{U} / \mathrm{ml})$; HOMA, the homeostasis model assessment; \%SD/chord, the recovery rate of the sum of $\mathrm{SD} /$ chord in the territories with occluded arteries from the acute to the chronic phase.

We evaluated the data of 24 subjects who underwent LVG in both the acute and chronic phase, and had either LAD or RCA territories supplied by the occluded artery. When compared between the acute and chronic phases, the $\mathrm{SD} /$ chord was decreased in the chronic phase and the other parameters were unchanged. The LVEDVI, LVESVI, and LVEF at 4 weeks after AMI (chronic phase) and the \%LVEDVI, \%LVESVI, and \%LVEF, were all similar in the 2 groups (Table 5). The \% SD/chord in the IGT group tended to be lower than in the NGT group, but not significantly (Table 5). There were no differences in the parameters shown in Tables 1-3 between any of the subjects or in those who were underwent LVG in both the acute and chronic phase (data not shown). The \%BMIPP TDS for IGT tended to be higher than for NGT in the subgroup of those who underwent LVG in both phases $(15 \%$ vs $24 \%$, NGT vs IGT, $\mathrm{p}=0.07$ ).

The IRI 120' positively correlated with the \%BMIPP TDS, but not with the \%Tl TDS (Fig 3), but neither the HOMA nor the glucose concentration (data not shown) correlated with either value. When the \%BMIPP TDS was made an independent variable and HOMA, BMI and IRI 120 ' became dependent variables based on multiple regression analysis, the goodness of fit was not significant. With respect to the RDS, the IRI 120' positively correlated with the \%BMIPP RDS $(\mathrm{r}=0.40, \mathrm{p}<0.05)$, but not with the \% $\mathrm{Tl}$ RDS. The HOMA did not correlate with \%BMIPP RDS.

The $\% \mathrm{SD} /$ chord inversely correlated with IRI 120 ' and HOMA (Fig 4), but the glucose concentration did not corre- 
late with the \%SD/chord (data not shown). When \% $\mathrm{SD} /$ chord was made an independent variable and HOMA, BMI and IRI 120' became dependent variables for multiple regression analysis, the goodness of fit was statistically significant $\left(\mathrm{r}^{2}=0.44, \mathrm{p}<0.01\right)$.

\section{Discussion}

Although there were no significant differences in the degree of recovery of Tl uptake, the rate of recovery of BMIPP uptake was significantly higher in the IGT group than in the NGT group. We found an inverse correlation between IR and the recovery of the RWM of the LV.

The present study demonstrated that the rate of recovery of the $\mathrm{SD} /$ chord in the territory supplied by the occluded artery inversely correlated with the degree of IR. It has been demonstrated previously that IR might contribute to the lower recovery of RWM after coronary artery bypass graft surgery ${ }^{26}$ and it has been reported that insulin-activated signaling pathways are protective in the ischemic heart through cell-survival signaling,27 suggesting that IR exacerbates the injury associated with myocardial infarction. Those results are consistent with the present findings. IR has been reported as promoting LV hypertrophy ${ }^{28}$ and it may influence the recovery of RWM ${ }^{29}$ We found no difference in the wall thickness of the non-infarcted LV on echocardiography (data not shown).

IR is accompanied not only by glucose intolerance, but also by abnormal lipid metabolism $3^{30}$ The main cause of IR is attributed to the blunted effect of insulin in the liver, skeletal muscle and adipose tissue; ${ }^{31}$ however, the pathophysiological significance of IR in the myocardium remains controversial ${ }^{14,15,32,33}$ A positive correlation between the IRI 120' and the recovery of BMIPP uptake was shown in the present study and a previous study found that the serum insulin concentration correlates inversely with the level of BMIPP uptake 34 These findings suggest that the myocardial uptake of BMIPP is influenced by the levels in the blood of several energy-providing substrates for the heart. However, it is not clear whether the higher \%BMIPP TDS in the IGT group was caused by a higher blood insulin concentration or by changes in the myocardium itself. It is possible that the decrease in myocardial glucose utilization because of IR causes an increase in utilization of fatty acids for energy metabolism in the myocardium 35

\section{Study Limitations}

With respect to the relationship between the recovery of RWM and IR, the relationship between the degree of insulin resistance and \% $\mathrm{SD} /$ chord was weak and therefore we cannot deny the possibility that the patients with IGT have a worsened insulin sensitivity because of the stress of the myocardial infarction (MI) and impaired LV wall motion.

Concerning the relationship between the recovery of BMIPP uptake and IR, \%BMIPP TDS was only related to IRI 120', not HOMA. It is possible that a decrease in the BMIPP defect score reflects increased late uptake of BMIPP in the infarct zone, a decrease in the non-ischemic zone, or a combination of the 2 . In the present study, absolute rates of BMIPP uptake were not measured and therefore it is difficult to differentiate between the possibilities. In addition, we could not demonstrate a statistical difference between the 2 groups in the BMIPP TDS in the chronic phase. We combined the data for the LAD, LCX and RCA territories for analysis, because of the small group of patients, so the sensitivity for detecting changes might be lower. It could be pointed out that the NGT group might have larger myocardial infarctions than the IGT group, although that was not statistically significant: the defect score of $\mathrm{Tl}$ in the acute phase was relatively higher in the NGT group than that in the IGT group. It might be attributed to the poor recovery of BMIPP uptake in the NGT group; however, the concentrations of creatine kinase (CK) and CKMB were similar in both groups and they are not in accordance with the greater recovery of RWM in the NGT group than the IGT group.

The small sample size in the present study precludes making any firm conclusions from the data. The findings could represent a chance effect and/or could be explained by differences in certain baseline characteristics of the population. Additional clinical and epidemiological studies are needed to determine more parameters to estimate RWM or fatty acid metabolism in the myocardium by using UCG and positron emission tomography (PET). We furthermore have to compare the parameters of this study among groups including a DM group. Although the region number and infarction area were not relevant for IR (data not shown), we have to take into consideration the contribution of several humoral factors, region area and region number to the recovery of BMIPP uptake and RWM during the phases of this study of AMI accompanied by IR.

IR influences several humoral factors, such as the sympathetic nervous system and renin-angiotensin-aldosterone system, in addition to the muscle blood flow, 36,37 and they may affect collateral flow and myocardial Ca-handling, contributing to the preconditioning of myocardium, even from before the occurrence of the AMI, and then influence the recovery of RWM of the LV after AMI. We also have to take into consideration the drugs administered at the occurrence of AMI, such as beta-blockers, angiotensinconverting enzyme inhibitors and statins, which also affect these humoral factors ${ }^{36}$ To evaluate these conditions in patients with IGT, but without the history of MI, is a further requirement.

\section{Conclusions}

In AMI patients who receive reperfusion therapy, IR accompanied by IGT can negatively influence the recovery of the RWM of the LV.

\section{References}

1. Alberti KG. The clinical implications of impaired glucose tolerance. Diabet Med 1996; 13: $927-937$.

2. International Collaborative Group. Asymptomatic Hyperglycemia and Coronary Heart Disease: A series of papers by the International Collaborative Group based on studies in fifteen populations. J Chron Dis 1979; 32: 681-837.

3. Fontbonne A, Eschwege E, Cambien F. Hypertriglyceridaemia as a risk factor of coronary heart disease mortality in subjects with impaired glucose tolerance or diabetes: Results from the 11-year follow-up of the Paris Prospective Study. Diabetologia 1989; 32: $300-304$.

4. Stengard JH, Tuomilehto J, Pekkanen J. Diabetes mellitus, impaired glucose tolerance and mortality among elderly men: The Finnish cohorts of the seven countries study. Diabetologia 1992; 35: 760765 .

5. Rewers M, Shetterly SM, Baxter J. Prevalence of coronary heart disease in subjects with normal and impaired glucose tolerance and non-insulin-dependent diabetes mellitus in a Biethnic Colorado Population: The San Luis Valley Diabetes Study. Am J Epidemiol 1992; 135: $1321-1330$. 
6. Rodrigues B, McNeill JH. The diabetic heart: Metabolic causes for the development of a cardiomyopathy. Cardiovasc Res 1992; 26: 913-922.

7. Stanley WC, Lopaschuk GD, McCormack JG. Regulation of energy substrate metabolism in the diabetic heart. Cardiovas Res 1997; 34: $25-33$.

8. Turpeinen AK, Knikka JT, Vanninen E. Abnormal myocardial kinetics of 123I-heptadecanoic acid in subjects with impaired glucose tolerance. Diabetologia 1997; 40: 541-549.

9. Turpeinen AK, Takala TO, Nuutila P, Axelin T, Luotolahti M, Haaparanta M, et al. Impaired free fatty acid uptake in skeletal muscle but not in myocardium in patients with impaired glucose tolerance: Studies with PET and 14(R,S)-[18F]fluoro-6-thia-heptadecanoic acid. Diabetes 1999; 48: 1245-1250.

10. Grines CL, Browne KF, Marco J, Rothbaum D, Stone GW, O'Keefe $\mathrm{J}$, et al. A comparison of immediate angioplasty with thrombolytic therapy for acute myocardial infarction: The Primary Angioplasty in Myocardial Infarction Study Group. N Engl J Med. 1993; 328: 673679.

11. Aronson D, Rayfield EJ, Chesebro JH. Mechanisms determining course and outcome of diabetic patients who have had acute myocardial infarction. Ann Intern Med 1997; 126: 296-306.

12. Zuanetti G, Latini R, the GISSI-3 investigators. Effect of the ACE inhibitor lisinopril on mortality in diabetic patients with acute myocardial infarction: Data from the GISSI-3 study. Circulation 1997; 96: $4239-4245$

13. Ito H, Taniyama Y, Iwakura K. Intravenous nicorandil can preserve microvascular integrity and myocardial viability in patients with reperfused anterior wall myocardial infarction. J Am Coll Cardiol 1999; 33: $654-660$

14. Gross GJ, Yao Z, Pieper GM. The ATP-regulated potassium channel in ischemia-reperfusion injury. Ann NY Acad Sci 1994; 723: 71-81.

15. Apstein CS. Glucose-insulin-potassium for acute myocardial infarction: Remarkable results from a new prospective, randomized trial. Circulation 1998; 98: 2223-2226.

16. The TIMI study group. The thrombolysis in myocardial infarction (TIMI) trial: Phase 1 findings. N Engl J Med 1984; 33: 523-530.

17. World Health Organization Study Group on Diabetes Mellitus. Technical report series 727. Geneva: WHO, 1985

18. Phillips DIW, Clark PM, Hales CN. Understanding oral glucose tolerance: Comparison of glucose or insulin measurements during the oral glucose tolerance test with specific measurements of insulin resistance and insulin secretion. Diabet Med 1994; 11: 286-292.

19. Matthews DR, Hosker JP, Rudenski AS. Homeostasis model assessment: Insulin resistance and beta-cell function from fasting plasma glucose and insulin concentrations in man. Diabetologia 1985; 28: 412-419.

20. Bonora E, Targher G, Alberiche M, Bonadonna RC, Saggiani F, Zenere MB, et al. Homeostasis model assessment closely mirrors the glucose clamp technique in the assessment of insulin sensitivity: Studies in subjects with various degrees of glucose tolerance and insulin sensitivity. Diabetes Care 2000; 23: 57-63.

21. Yamagishi H, Akioka K, Shirai N, Yoshiyama M, Teragaki M, Takeuchi K, et al. Effects of smoking on myocardial injury in patients with conservatively treated acute myocardial infarction: A study with resting ${ }^{123}$ I-15-iodophenyl 3-methyl pentadecanoic acid/201Tl myocardial single photon emission computed tomography. Circ J 2001; 65: 769-774.

22. Sandler H, Dodge HT. The use of single plane angiocardiograms for the calculation of left ventricular volume in man. Am Heart J 1968; 75: $325-334$.

23. Sheehan FH, Bolson EL, Dodge HT. Advantages and applications of the centerline method for characterizing regional ventricular function. Circulation 1986; 74: 293-305.

24. Matsuda M, DeFronzo R. Insulin sensitivity indices obtained from oral glucose tolerance testing. Diabetes Care 1999; 22: 1462-1470.

25. Yanagisawa H, Chikamori T, Tanaka N, Hatano T, Morishima T, Hida S, et al. Correlation between thallium-201 myocardial perfusion defects and the functional severity of coronary artery stenosis as assessed by pressure-derived myocardial fractional flow reserve. Circ J 2002; 66: 1105-1109.

26. Marinho NVS, Keogh BE, Costa DC. Pathophysiology of chronic left ventricular dysfunction: New insights from the measurement of absolute myocardial blood flow and glucose utilization. Circulation 1996; 93: $737-744$.

27. Jonassen AK, Sack MN, Mjos OD, Yellon DM. Myocardial protection by insulin at reperfusion requires early administration and is mediated via Akt and p70s6 kinase cell-survival signaling. Circ Res 2001; 89: $1191-1198$.

28. Lind L, Andersson PE, Andren B. Left ventricular hypertrophy in hypertension is associated with the insulin resistance metabolic syndrome. J Hypertens 1995; 13: 433-438.

29. Koren MJ, Devereux RB, Casale PN. Relation of left ventricular mass and geometry to morbidity and mortality in uncomplicated essential hypertension. Ann Intern Med 1991; 114: 345-352.

30. Frayn KN. Insulin resistance and lipid metabolism. Curr Opin Lipidol 1993; 4: 197-204.

31. Nuutila P, Knuuti J, Ruotsalainen U. Insulin resistance is localized to skeletal but not heart muscle in type 1 diabetes. Am J Physiol 1993; 264: E756-E762.

32. Paternostro G, Camici PG, Lammerstma AA. Cardiac and skeletal muscle insulin resistance in patients with coronary heart disease: A study with positron emission tomography. J Clin Invest 1996; 98: 2094-2099.

33. Voipio-pulkki LM, Nuutila P, Knuuti J. Heart and skeletal muscle glucose disposal in type 2 diabetic patients as determined by positron emission tomography. J Nucl Med 1993; 34: 2064-2067.

34. Kurata C, Wakabayashi Y, Shouda S. Influence of blood substrate levels on myocardial kinetics of iodine-123-BMIPP. J Nucl Med 1997; 38: 1079-1084.

35. Nuutila P, Koivisto VA, Knuuti J. Glucose-free fatty acid cycle operates in human heart and skeletal muscle in vivo. J Clin Invest 1992; 89: $1767-1774$

36. Ginsberg HN. Treatment for patients with the metabolic syndrome. Am J Cardiol 2003; 91: 29E-39E.

37. Scherrer U, Sartori C. Insulin as a vascular and sympathoexcitatory hormone: Implications for blood pressure regulation, insulin sensitivity, and cardiovascular morbidity. Circulation 1997; 96: 41044113. 